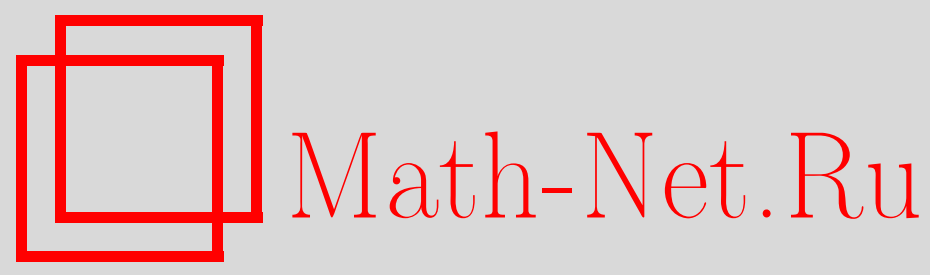

В. П. Маслов, О высокотемпературной сверхпроводимости, Матем. заметки, 1999, том 66, выпуск 5, 706-722

DOI: https://doi.org/10.4213/mzm1215

Использование Общероссийского математического портала Math-Net.Ru подразумевает, что вы прочитали и согласны с пользовательским соглашением http://www . mathnet.ru/rus/agreement

Параметры загрузки:

IP: 3.91 .87 .62

26 апреля 2023 г., 13:43:42

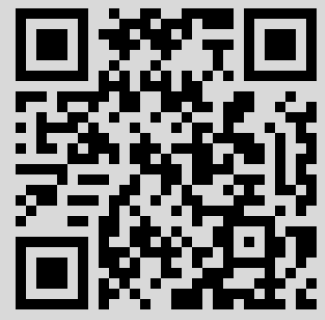




\section{О ВЫСОКОТЕМПЕРАТУРНОЙ СВЕРХПРОВОДИМОСТИ}

\section{В.П. Маслов}

Рассматриваются модели электронно-фононных взаимодействий при условии сильной связи, а также известная модель Хаббарда. Предъявляется асимптотика при числе электронов, стремящемся к бесконечности, позволяющая определить температуру фазового перехода из сверхпроводящего состояния в нормальное, а также скачок теплоемкости.

Библиограбфия: 7 названий.

В настоящее время существуют, по-видимому, две возможные концепции высокотемпературной сверхпроводимости. Во-первых, это теория сильного взаимодействия электронов с фононами и, возможно, электромагнитным полем; во-вторых, решетчатая модель Хаббарда. Мы рассмотрим с математических позищий оба эти случая.

Рассматриваемая задача имеет два больших параметра: параметр, описьвающий сильное взаимодействие, и параметр, отвечаюший большому числу фермионов. Отметим вначале, что приведенные ниже выкладки дают решение проблемы при условии равномерности приведенных асимптотик, проведенных по одному из параметров относительно другого параметра. Эти оценки столь громоздки, что в настоящей статье мы их проводить не будем, а проведем лишь сами выкладки. Прежде всего отметим, что приближение сильного взаимодействия при отсутствии внешнего поля равносильно асимптотике при $\bar{h} \rightarrow 0$. В случае модели Хаббарда эта асимптотика равносильна асимптотике по большому числу узлов решетки.

Поэтому мы приведем асимптотику при $\bar{h} \rightarrow 0$ для общей вторично квантованной задачи при условии, что число $N$ фермионов сохраняется. Мы применим метод операторнозначного символа, разработанньй в [1].

Рассмотрим систему бозонов и фермионов со спином $1 / 2$. Пусть гамильтониан системы в представлении вторичного квантования имеет следующий вид:

$$
\begin{aligned}
\widehat{\mathcal{H}}= & \sum_{s=1}^{2} \int d x \widehat{\psi}_{s}^{+}(x) H_{0}\left(\stackrel{2}{x},-i \hbar \frac{\partial}{\partial x} ; \hbar\right) \widehat{\psi}_{s}^{-}(x)+\sum_{\alpha=1}^{N_{B}} \sum_{\vec{n}} \Omega_{\alpha}\left(\frac{2 \pi \vec{n}}{L}\right) \widehat{b}_{\alpha}^{+}(\vec{n}) \widehat{b}_{\alpha}^{-}(\vec{n}) \\
& +\sum_{s=1}^{2} \sum_{\alpha=1}^{N_{B}} \sum_{\vec{n}} \int d x\left(\widehat{b}_{\alpha}^{+}(\vec{n}) \widehat{\psi}_{s}^{+}(x) f_{\alpha}\left(\frac{2 \pi}{L} \vec{n} ; \stackrel{2}{x},-i \hbar \frac{\partial}{\partial x} ; \hbar\right) \widehat{\psi}_{s}^{-}(x)\right. \\
& \left.+\widehat{b}_{\alpha}^{-}(\vec{n}) \widehat{\psi}_{s}^{+}(x) f_{\alpha}^{*}\left(\frac{2 \pi}{L} \vec{n} ; \stackrel{1}{x},-i \hbar \frac{\partial}{\partial x} ; \hbar\right) \widehat{\psi}_{s}^{-}(x)\right)
\end{aligned}
$$




$$
\begin{aligned}
& +\sum_{s=1}^{2} \sum_{\alpha=1}^{N_{B}} \sum_{\beta=1}^{N_{B}} \sum_{\vec{n}} \sum_{\vec{n}^{\prime}} \int d x \widehat{\psi}_{s}^{+}(x)\left(A_{\alpha \beta}\left(\frac{2 \pi}{L} \vec{n} ; \frac{2 \pi}{L} \vec{n}^{\prime} ; \stackrel{2}{x},-i \hbar \frac{\partial}{\partial x} ; \hbar\right) \widehat{b}_{\alpha}^{+}(\vec{n}) \widehat{b}_{\beta}^{-}\left(\vec{n}^{\prime}\right)\right. \\
& +B_{\alpha \beta}\left(\frac{2 \pi}{L} \vec{n} ; \frac{2 \pi}{L} \vec{n}^{\prime} ; 2,-i \hbar \frac{\partial}{\partial x} ; \hbar\right) \widehat{b}_{\alpha}^{+}(\vec{n}) \widehat{b}_{\beta}^{+}\left(\vec{n}^{\prime}\right) \\
& \left.+B_{\alpha \beta}^{*}\left(\frac{2 \pi}{L} \vec{n} ; \frac{2 \pi}{L} \vec{n}^{\prime} ; \frac{1}{x},-i \hbar \frac{\partial}{\partial x} ; \hbar\right) \widehat{b}_{\beta}^{-}\left(\vec{n}^{\prime}\right) \widehat{b}_{\alpha}^{-}(\vec{n})\right) \widehat{\psi}_{s}^{-}(x) \\
& +\frac{1}{2} \sum_{s=1}^{2} \sum_{s^{\prime}=1}^{2} \iint d x d x^{\prime} \widehat{\psi}_{s}^{+}(x) \widehat{\psi}_{s^{\prime}}^{+}\left(x^{\prime}\right) V\left(\stackrel{2}{x},-i \hbar \frac{\partial}{\partial x} ; x^{\prime},-i \hbar \frac{\partial}{\partial x^{\prime}} ; \hbar\right) \widehat{\psi}_{s^{\prime}}^{-}\left(x^{\prime}\right) \widehat{\psi}_{s}^{-}(x)
\end{aligned}
$$

где $x \in \mathbb{R}, \vec{n}=\left(n_{1}, n_{2}, n_{3}\right), n_{j} \in \mathbb{Z}, j=1,2,3, \sum_{\vec{n}}=\sum_{n_{1}=-\infty}^{+\infty} \sum_{n_{2}=-\infty}^{+\infty} \sum_{n_{3}=-\infty}^{+\infty}$, $\hbar>0$ - постоянная Планка, $N_{B}$ - число типов бозонов, $L>0$ - длина стороны трехмерного тора, на котором находится система; $\Omega_{\alpha}(q) \in C^{\infty}\left(\mathbb{R}^{3}\right)$ при всех $\alpha=1, \ldots, N_{B}$, $H_{0}(x, p ; \hbar) \in C^{\infty}\left(\mathbb{R}^{7}\right)$ такая функция, что $\hbar$-псевдодифференциальный оператор [1] $H(\stackrel{2}{x},-i \hbar \partial / \partial x ; \hbar)$ самосопряжен; $V\left(x_{1}, p_{1} ; x_{2}, p_{2} ; \hbar\right) \in C^{\infty}\left(\mathbb{R}^{13}\right)$ такая функция, что $V\left(x_{1}, p_{1} ; x_{2}, p_{2} ; \hbar\right)=V\left(x_{2}, p_{2} ; x_{1}, p_{1} ; \hbar\right)$ и $V\left(\stackrel{2}{x_{1}},-i \hbar \partial \stackrel{1}{2} / \partial x_{1} ; \stackrel{2}{x_{2}},-i \hbar \partial \stackrel{1}{\partial} / \partial x_{2} ; \hbar\right)-$ самосопряженньй оператор; числа над операторами обозначают порядок их действий [1]; $f_{\alpha}(q ; x, p ; \hbar) \in C^{\infty}\left(\mathbb{R}^{10}\right), A_{\alpha \beta}\left(q, q^{\prime} ; x, p ; \hbar\right) \in C^{\infty}\left(\mathbb{R}^{13}\right)$, и $B_{\alpha \beta}\left(q, q^{\prime} ; x, p ; \hbar\right) \in C^{\infty}\left(\mathbb{R}^{13}\right)$ для всех $\alpha, \beta=1, \ldots, N_{B}$ функции такие, что $B_{\alpha \beta}\left(q, q^{\prime} ; x, p ; \hbar\right)=B_{\beta \alpha}\left(q, q^{\prime} ; x, p ; \hbar\right)$ и $A_{\alpha \beta}\left(q, q^{\prime} ; \stackrel{2}{x},-i \hbar \partial / \partial x ; \hbar\right)=A_{\beta \alpha}^{*}\left(q, q^{\prime} ; \stackrel{1}{x},-i \hbar \partial / 2 x ; \hbar\right)$; операторы $\widehat{\psi}_{s}^{ \pm}(x)$ удовлетворяют следуюшим антикоммутационным соотношениям

$$
\left\{\widehat{\psi}_{s}^{-}(x), \widehat{\psi}_{s^{\prime}}^{+}\left(x^{\prime}\right)\right\}=\delta_{s s^{\prime}} \delta\left(x-x^{\prime}\right), \quad\left\{\widehat{\psi}_{s}^{ \pm}(x), \widehat{\psi}_{s^{\prime}}^{ \pm}\left(x^{\prime}\right)\right\}=0
$$

а операторы $\widehat{b}_{\alpha}^{ \pm}(\vec{n})$ удовлетворяют коммутационньг соотношениям вида

$$
\begin{gathered}
{\left[\widehat{b}_{\alpha}^{-}(\vec{n}), \widehat{b}_{\beta}^{+}\left(\vec{n}^{\prime}\right)\right]=\delta_{\alpha \beta} \delta_{\vec{n}, \vec{n}^{\prime}}, \quad\left[\widehat{b}_{\alpha}^{ \pm}(\vec{n}), \widehat{b}_{\beta}^{ \pm}\left(\vec{n}^{\prime}\right)\right]=0} \\
{\left[\widehat{\psi}_{s}^{\mp}(x), \widehat{b}_{\alpha}^{ \pm}(\vec{n})\right]=\left[\widehat{\psi}_{s}^{ \pm}(x), \widehat{b}_{\alpha}^{ \pm}(\vec{n})\right]=0}
\end{gathered}
$$

где $\delta_{r s}=\left\{\begin{array}{l}1, r=s, \\ 0, r \neq s\end{array}\right.$ - символ Кронекера для всех $r, s \in \mathbb{Z}, \delta_{\vec{n}, \vec{n}^{\prime}}=\delta_{n_{1}, n_{1}^{\prime}} \delta_{n_{2}, n_{2}^{\prime}} \delta_{n_{3}, n_{3}^{\prime}}$.

Пусть число фермионов в системе равно $N$. В этом случае гамильтониану (1) отвечает следующее уравнение Шрёдингера:

$$
\begin{gathered}
\left\{\sum_{j=1}^{N} H_{0}\left(\stackrel{2}{x}_{j},-i \hbar \frac{\partial}{\partial x_{j}} ; \hbar\right)+\frac{1}{2} \sum_{j=1}^{N} \sum_{k=1, k \neq j}^{N} V\left(\stackrel{2}{x}_{j},-i \hbar \frac{\partial}{\partial x_{j}} ; \stackrel{2}{x}_{k},-i \hbar \frac{\partial}{\partial x_{k}} ; \hbar\right)\right. \\
+\frac{1}{2} \sum_{\alpha=1}^{N_{B}} \sum_{\vec{n}} \Omega_{\alpha}\left(\frac{2 \pi}{L} \vec{n}\right)\left(Q_{\alpha, \vec{n}}-\frac{\partial}{\partial Q_{\alpha, \vec{n}}}\right)\left(Q_{\alpha, \vec{n}}+\frac{\partial}{\partial Q_{\alpha, \vec{n}}}\right)
\end{gathered}
$$




$$
\begin{aligned}
& +\frac{1}{\sqrt{2}} \sum_{\alpha=1}^{N_{B}} \sum_{\vec{n}} \sum_{j=1}^{N}\left(\left(Q_{\alpha, \vec{n}}-\frac{\partial}{\partial Q_{\alpha, \vec{n}}}\right) f_{\alpha}\left(\frac{2 \pi}{L} \vec{n} ; \stackrel{2}{x}_{j},-i \hbar \frac{\partial}{\partial x_{j}} ; \hbar\right)\right. \\
& \left.+\left(Q_{\alpha, \vec{n}}-\frac{\partial}{\partial Q_{\alpha, \vec{n}}}\right) f_{\alpha}^{*}\left(\frac{2 \pi}{L} \vec{n} ; x_{j},-i \hbar \frac{\partial}{\partial x_{j}} ; \hbar\right)\right) \\
& +\frac{1}{2} \sum_{\alpha=1}^{N_{B}} \sum_{\beta=1}^{N_{B}} \sum_{\vec{n}} \sum_{\vec{n}} \sum_{j=1}^{N}\left(A_{\alpha \beta}\left(\frac{2 \pi}{L} \vec{n} ; \frac{2 \pi}{L} \vec{n}^{\prime} ; \stackrel{2}{x}_{j},-i \hbar \frac{\partial}{\partial x_{j}} ; \hbar\right)\left(Q_{\alpha, \vec{n}}-\frac{\partial}{\partial Q_{\alpha, \vec{n}}}\right)\right. \\
& \times\left(Q_{\beta, \vec{n}^{\prime}}+\frac{\partial}{\partial Q_{\beta, \vec{n}^{\prime}}}\right) \\
& +B_{\alpha \beta}\left(\frac{2 \pi}{L} \vec{n} ; \frac{2 \pi}{L} \vec{n}^{\prime} ; \stackrel{2}{x}_{j},-i \hbar \frac{\partial}{\partial x_{j}} ; \hbar\right)\left(Q_{\alpha, \vec{n}^{\prime}}-\frac{\partial}{\partial Q_{\alpha, \vec{n}}}\right)\left(Q_{\beta, \vec{n}^{\prime}}-\frac{\partial}{\partial Q_{\beta, \vec{n}^{\prime}}}\right) \\
& \left.+B_{\alpha \beta}^{*}\left(\frac{2 \pi}{L} \vec{n} ; \frac{2 \pi}{L} \vec{n}^{\prime} ; \stackrel{1}{x}_{j},-i \hbar \frac{\partial}{\partial x_{j}} ; \hbar\right)\left(Q_{\beta, \vec{n}^{\prime}}+\frac{\partial}{\partial Q_{\beta, \vec{n}^{\prime}}}\right)\left(Q_{\alpha, \vec{n}}+\frac{\partial}{\partial Q_{\alpha, \vec{n}}}\right)\right) \\
& \times \Phi\left(x_{1}, s_{1} ; \ldots ; x_{N}, s_{N} ; Q ; t\right) \\
& =i \hbar \frac{\partial}{\partial t} \Phi\left(x_{1}, s_{1} ; \ldots ; x_{N}, s_{N} ; Q ; t\right),
\end{aligned}
$$

где $Q_{\alpha, \vec{n}} \in \mathbb{R}$ при всех $\alpha=1, \ldots, N_{B}$ и при всех $\vec{n}$ и функция $\Phi\left(x_{1}, s_{1} ; \ldots ; x_{N}, s_{N} ; Q ; t\right)$ антисимметрична относительно перестановки $x_{j}, s_{j}$ и $x_{k}, s_{k}$ при всех $j, k=1, \ldots, N$.

Для нахождения асимптотических решений уравнения (2) в пределе при $\hbar \rightarrow 0$, необходимо решить следуюшее уравнение [1]

$$
\begin{aligned}
& \left\{\sum_{j=1}^{N} H_{0}\left(x_{j}, p_{j}\right)+\sum_{\alpha=1}^{N_{B}} \sum_{\vec{n}} \frac{1}{2} \Omega_{\alpha}\left(\frac{2 \pi}{L} \vec{n}\right)\left(Q_{\alpha, \vec{n}}-\frac{\partial}{\partial Q_{\alpha, \vec{n}}}\right)\left(Q_{\alpha, \vec{n}}+\frac{\partial}{\partial Q_{\alpha, \vec{n}}}\right)\right. \\
& +\frac{1}{\sqrt{2}} \sum_{\alpha=1}^{N_{B}} \sum_{\vec{n}} \sum_{j=1}^{N}\left(f_{\alpha}\left(\frac{2 \pi}{L} \vec{n} ; x_{j}, p_{j}\right)\left(Q_{\alpha, \vec{n}}-\frac{\partial}{\partial Q_{\alpha, \vec{n}}}\right)\right. \\
& \left.+f_{\alpha}^{*}\left(\frac{2 \pi}{L} \vec{n} ; x_{j}, p_{j}\right)\left(Q_{\alpha, \vec{n}}+\frac{\partial}{\partial Q_{\alpha, \vec{n}}}\right)\right) \\
& +\frac{1}{2} \sum_{\alpha=1}^{N_{B}} \sum_{\beta=1}^{N_{B}} \sum_{\vec{n}} \sum_{\vec{n}} \sum_{j=1}^{N}\left(A_{\alpha \beta}\left(\frac{2 \pi}{L} \vec{n} ; \frac{2 \pi}{L} \vec{n}^{\prime} ; x_{j}, p_{j}\right)\right. \\
& \times\left(Q_{\alpha, \vec{n}}-\frac{\partial}{\partial Q_{\alpha, \vec{n}}}\right)\left(Q_{\beta, \vec{n}^{\prime}}+\frac{\partial}{\partial Q_{\beta, \vec{n}^{\prime}}}\right) \\
& +B_{\alpha \beta}\left(\frac{2 \pi}{L} \vec{n} ; \frac{2 \pi}{L} \vec{n}^{\prime} ; x_{j}, p_{j}\right)\left(Q_{\alpha, \vec{n}}-\frac{\partial}{\partial Q_{\alpha, \vec{n}}}\right)\left(Q_{\beta, \vec{n}^{\prime}}-\frac{\partial}{\partial Q_{\beta, \vec{n}^{\prime}}}\right) \\
& \left.+B_{\alpha \beta}^{*}\left(\frac{2 \pi}{L} \vec{n} ; \frac{2 \pi}{L} \vec{n}^{\prime} ; x_{j}, p_{j}\right)\left(Q_{\beta, \vec{n}^{\prime}}+\frac{\partial}{\partial Q_{\beta, \vec{n}^{\prime}}}\right)\left(Q_{\alpha, \vec{n}}+\frac{\partial}{\partial Q_{\alpha, \vec{n}}}\right)\right) \\
& \left.+\frac{1}{2} \sum_{j=1}^{N} \sum_{k=1, k \neq j}^{N} V\left(x_{j}, p_{j} ; x_{k}, p_{k}\right)\right\} \chi\left(x_{1}, p_{1} ; \ldots ; x_{N}, p_{N} ; Q\right)
\end{aligned}
$$




$$
=\lambda\left(x_{1}, p_{1} ; \ldots ; x_{N}, p_{N}\right) \chi\left(x_{1}, p_{1} ; \ldots ; x_{N}, p_{N} ; Q\right),
$$

где $\chi\left(x_{1}, p_{1} ; \ldots ; x_{N}, p_{N} ; Q\right)$ и $\lambda\left(x_{1}, p_{1} ; \ldots ; x_{N}, p_{N}\right)$ зависят от $x_{j}, p_{j}, j=1, \ldots, N$, как от параметров, $H_{0}(x, p)=H_{0}(x, p ; 0), V\left(x_{1}, p_{1} ; x_{2}, p_{2}\right)=V\left(x_{1}, p_{1} ; x_{2}, p_{2} ; 0\right)$, $f_{\alpha}(q ; x, p)=f_{\alpha}(q ; x, p ; 0), A_{\alpha \beta}\left(q, q^{\prime} ; x, p\right)=A_{\alpha \beta}\left(q, q^{\prime} ; x, p ; 0\right)$, и $B_{\alpha \beta}\left(q, q^{\prime} ; x, p\right)=$ $B_{\alpha \beta}\left(q, q^{\prime} ; x, p ; 0\right)$. В силу определения эти функции удовлетворяют соотношениям $H_{0}(x, p)=H_{0}^{*}(x, p), V\left(x_{1}, p_{1} ; x_{2}, p_{2}\right)=V^{*}\left(x_{1}, p_{1} ; x_{2}, p_{2}\right), A_{\alpha \beta}\left(q, q^{\prime} ; x, p\right)=$ $A_{\beta \alpha}^{*}\left(q, q^{\prime} ; x, p\right)$, и $B_{\alpha \beta}\left(q, q^{\prime} ; x, p\right)=B_{\beta \alpha}\left(q, q^{\prime} ; x, p\right)$. Собственные значения уравнения (3) $\lambda\left(x_{1}, p_{1} ; \ldots ; x_{N}, p_{N}\right)$ называются [1] термами. Они играют роль классических гамильтонианов для системы $N$ частиц. В дальнейшем будем считать, что кратность термов постоянна при всех $x, p$.

Пусть $\chi_{\mu}\left(x_{1}, p_{1} ; \ldots ; x_{N}, p_{N} ; Q\right), \mu=1, \ldots, K,-$ решения уравнения (3), отвечающие терму $\lambda\left(x_{1}, p_{1} ; \ldots ; x_{N}, p_{N}\right)$, где $K$ кратность этого терма. Будем считать, что

$$
\int \cdots \int \prod_{\alpha=1}^{N_{B}} \prod_{n_{1}=-\infty}^{+\infty} \prod_{n_{2}=-\infty}^{+\infty} \prod_{n_{3}=-\infty}^{+\infty} d Q_{\alpha, \vec{n}} \chi_{\nu}^{*}\left(x_{1}, p_{1} ; \ldots ; x_{N}, p_{N} ; Q\right)=\delta_{\mu \nu}
$$

Согласно [2], терму $\lambda\left(x_{1}, p_{1} ; \ldots ; x_{N}, p_{N}\right)$ отвечает следующая система уравнений:

$$
\begin{aligned}
\frac{\partial u_{\nu}}{\partial t}\left(x_{1}, p_{1} ; \ldots ; x_{N}, p_{N} ; t\right)+\sum_{j=1}^{N}\left(\frac{\partial u_{\nu}}{\partial t}\left(x_{1}, p_{1} ; \ldots ; x_{N}, p_{N} ; t\right) \frac{\partial \lambda}{\partial p_{j}}\left(x_{1}, p_{1} ; \ldots ; x_{N}, p_{N}\right)\right. \\
\left.\quad-\frac{\partial u_{\nu}}{\partial \partial p_{j}}\left(x_{1}, p_{1} ; \ldots ; x_{N}, p_{N} ; t\right) \frac{\partial \lambda}{\partial x_{j}}\left(x_{1}, p_{1} ; \ldots ; x_{N}, p_{N}\right)\right) \\
\quad+\sum_{\mu=1}^{K} G_{\nu \mu}\left(x_{1}, p_{1} ; \ldots ; x_{N}, p_{N}\right) u_{\mu}\left(x_{1}, p_{1} ; \ldots ; x_{N}, p_{N} ; t\right)=0, \quad \nu=1, \ldots, K
\end{aligned}
$$

где $G_{\nu \mu}\left(x_{1}, p_{1} ; \ldots ; x_{N}, p_{N}\right)$ имеет вид

$$
\begin{aligned}
G_{\nu \mu}\left(x_{1}, p_{1} ; \ldots ; x_{N}, p_{N}\right) & \int \ldots \int \prod_{\alpha=1}^{N_{B}} \prod_{n_{1}=-\infty}^{+\infty} \prod_{n_{2}=-\infty}^{+\infty} \prod_{n_{3}=-\infty}^{+\infty} d Q_{\alpha, \vec{n}} \chi_{\nu}^{*}\left(x_{1}, p_{1} ; \ldots ; x_{N}, p_{N}\right) \\
= & \left(-\sum_{j=1}^{N} \frac{\partial \lambda}{\partial x_{j}}\left(x_{1}, p_{1} ; \ldots ; x_{N}, p_{N}\right) \frac{\partial}{\partial p_{j}}\right. \\
& +\sum_{j=1}^{N}\left(\frac{\partial H_{0}}{\partial p_{j}}\left(x_{j}, p_{j}\right)+\frac{1}{\sqrt{2}} \sum_{\beta=1}^{N_{B}} \sum_{\vec{n}^{\prime}}\left(\frac{\partial f_{\beta}}{\partial p_{j}}\left(\frac{2 \pi}{L} \vec{n}^{\prime} ; x_{j}, p_{j}\right)\left(Q_{\beta, \vec{n}^{\prime}}-\frac{\partial}{\partial Q_{\beta, \vec{n}^{\prime}}}\right)\right.\right. \\
& \left.+\frac{\partial f_{\beta}^{*}}{\partial p_{j}}\left(\frac{2 \pi}{L} \vec{n}^{\prime} ; x_{j}, p_{j}\right)\left(Q_{\beta, \vec{n}^{\prime}}+\frac{\partial}{\partial Q_{\beta, \vec{n}^{\prime}}}\right)\right) \\
& +\frac{1}{2} \sum_{\beta=1}^{N_{B}} \sum_{\gamma=1}^{N_{B}} \sum_{\vec{n}^{\prime}} \sum_{\vec{n}^{\prime \prime}}\left(\frac{\partial A_{\beta \gamma}}{\partial p_{j}}\left(\frac{2 \pi}{L} \vec{n}^{\prime}, \frac{2 \pi}{L} \vec{n}^{\prime \prime} ; x_{j}, p_{j}\right)\right.
\end{aligned}
$$




$$
\begin{aligned}
& \times\left(Q_{\beta, \vec{n}^{\prime}}-\frac{\partial}{\partial Q_{\beta, \vec{n}^{\prime}}}\right)\left(Q_{\gamma, \vec{n}^{\prime \prime}}-\frac{\partial}{\partial Q_{\gamma, \vec{n}^{\prime \prime}}}\right) \\
& +\frac{\partial B_{\beta \gamma}}{\partial p_{j}}\left(\frac{2 \pi}{L} \vec{n}^{\prime}, \frac{2 \pi}{L} \vec{n}^{\prime \prime} ; x_{j}, p_{j}\right)\left(Q_{\beta, \vec{n}^{\prime}}-\frac{\partial}{\partial Q_{\beta, \vec{n}^{\prime}}}\right)\left(Q_{\gamma, \vec{n}^{\prime \prime}}+\frac{\partial}{\partial Q_{\gamma, \vec{n}^{\prime \prime}}}\right) \\
& \left.+\frac{\partial B_{\beta \gamma}^{*}}{\partial p_{j}}\left(\frac{2 \pi}{L} \vec{n}^{\prime}, \frac{2 \pi}{L} \vec{n}^{\prime \prime} ; x_{j}, p_{j}\right)\left(Q_{\gamma, \vec{n}^{\prime \prime}}+\frac{\partial}{\partial Q_{\gamma, \vec{n}^{\prime \prime}}}\right)\left(Q_{\beta, \vec{n}^{\prime}}+\frac{\partial}{\partial Q_{\beta, \vec{n}^{\prime}}}\right)\right) \\
& +\frac{1}{2} \sum_{k=1, k \neq j}\left(\frac{\partial V}{\partial p_{j}}\left(x_{j}, p_{j} ; x_{k}, p_{k}\right)+\frac{\partial V}{\partial p_{j}}\left(x_{k}, p_{k} ; x_{j}, p_{j}\right)\right) \frac{\partial}{\partial x_{j}} \\
& -\frac{1}{2} \sum_{j=1}^{N} \frac{\partial^{2} \lambda}{\partial x_{j} \partial p_{j}}\left(x_{1}, p_{1} ; \ldots ; x_{N}, p_{n}\right) \\
& +i\left(\sum_{j=1}^{N} \frac{\partial H_{0}}{\partial \hbar}\left(x_{j}, p_{j} ; 0\right)+\frac{1}{2} \sum_{j=1}^{N} \sum_{k=1, k \neq j}^{N} \frac{\partial V}{\partial \hbar}\left(x_{j}, p_{j} ; x_{k}, p_{k} ; 0\right)\right. \\
& +\frac{1}{\sqrt{2}} \sum_{\beta=1}^{N_{B}} \sum_{\vec{n}^{\prime}} \sum_{j=1}^{N}\left(\left(Q_{\beta, \vec{n}^{\prime}}-\frac{\partial}{\partial Q_{\beta, \vec{n}^{\prime}}}\right) \frac{\partial f_{\alpha}}{\partial \hbar}\left(\frac{2 \pi}{L} \vec{n}^{\prime} ; x_{j}, p_{j} ; 0\right)\right. \\
& +\left(Q_{\beta, \vec{n}^{\prime}}+\frac{\partial}{\partial Q_{\beta, \vec{n}^{\prime}}}\right) \frac{\partial f_{\alpha}^{*}}{\partial \hbar}\left(\frac{2 \pi}{L} \vec{n}^{\prime} ; x_{j}, p_{j} ; 0\right) \\
& \left.-i\left(Q_{\beta, \vec{n}^{\prime}}+\frac{\partial}{\partial Q_{\beta, \vec{n}^{\prime}}}\right) \frac{\partial^{2} f_{\alpha}^{*}}{\partial x_{j} \partial p_{j}}\left(\frac{2 \pi}{L} \vec{n}^{\prime} ; x_{j}, p_{j} ; 0\right)\right) \\
& +\frac{1}{2} \sum_{\beta=1}^{N_{B}} \sum_{\gamma=1}^{N_{B}} \sum_{\vec{n}^{\prime}} \sum_{\vec{n}^{\prime \prime}} \sum_{j=1}^{N}\left(\frac{\partial A_{\beta \gamma}}{\partial \hbar}\left(\frac{2 \pi}{L} \vec{n}^{\prime}, \frac{2 \pi}{L} \vec{n}^{\prime \prime} ; x_{j}, p_{j} ; 0\right)\left(Q_{\beta, \vec{n}^{\prime}}-\frac{\partial}{\partial Q_{\beta, \vec{n}^{\prime}}}\right)\right. \\
& \times\left(Q_{\beta, \vec{n}^{\prime \prime}}+\frac{\partial}{\partial Q_{\beta, \vec{n}^{\prime \prime}}}\right) \\
& +\frac{\partial B_{\beta \gamma}}{\partial \hbar}\left(\frac{2 \pi}{L} \vec{n}^{\prime}, \frac{2 \pi}{L} \vec{n}^{\prime \prime} ; x_{j}, p_{j} ; 0\right)\left(Q_{\beta, \vec{n}^{\prime}}-\frac{\partial}{\partial Q_{\beta, \vec{n}^{\prime}}}\right)\left(Q_{\beta, \vec{n}^{\prime \prime}}-\frac{\partial}{\partial Q_{\beta, \vec{n}^{\prime \prime}}}\right) \\
& +\frac{\partial B_{\beta \gamma}^{*}}{\partial \hbar}\left(\frac{2 \pi}{L} \vec{n}^{\prime}, \frac{2 \pi}{L} \vec{n}^{\prime \prime} ; x_{j}, p_{j} ; 0\right)\left(Q_{\beta, \vec{n}^{\prime \prime}}+\frac{\partial}{\partial Q_{\beta, \vec{n}^{\prime \prime}}}\right)\left(Q_{\beta, \vec{n}^{\prime}}+\frac{\partial}{\partial Q_{\beta, \vec{n}^{\prime}}}\right) \\
& \left.\left.-i \frac{\partial^{2} B_{\beta \gamma}^{*}}{\partial x_{j} p_{j}}\left(\frac{2 \pi}{L} \vec{n}^{\prime}, \frac{2 \pi}{L} \vec{n}^{\prime \prime} ; x_{j}, p_{j} ; 0\right)\left(Q_{\beta, \vec{n}^{\prime \prime}}+\frac{\partial}{\partial Q_{\beta, \vec{n}^{\prime \prime}}}\right)\left(Q_{\beta, \vec{n}^{\prime}}+\frac{\partial}{\partial Q_{\beta, \vec{n}^{\prime}}}\right)\right)\right) \\
& \times \chi_{\mu}\left(x_{1}, p_{1} ; \ldots ; x_{N}, p_{N} ; 0\right) \text {. }
\end{aligned}
$$

В работе [1] приведены решения уравнения вида:

$$
\begin{aligned}
\frac{1}{2} \sum_{j=1}^{M} \sum_{k=1}^{M} & \left(A_{j k}\left(q_{j}-\frac{\partial}{\partial q_{j}}\right)\left(q_{k}+\frac{\partial}{\partial q_{k}}\right)+D_{j k}\left(q_{j}-\frac{\partial}{\partial q_{j}}\right)\left(q_{k}-\frac{\partial}{\partial q_{k}}\right)\right. \\
& \left.+D_{j k}^{*}\left(q_{j}+\frac{\partial}{\partial q_{j}}\right)\left(q_{k}+\frac{\partial}{\partial q_{k}}\right)\right) \varphi\left(q_{1}, \ldots, q_{M}\right) \\
& +\frac{1}{\sqrt{2}} \sum_{j=1}^{M}\left(f_{j}\left(q_{j}-\frac{\partial}{\partial q_{j}}\right)+f_{j}^{*}\left(q_{j}+\frac{\partial}{\partial q_{j}}\right)\right) \varphi\left(q_{1}, \ldots, q_{M}\right)=\lambda \varphi\left(q_{1}, \ldots, q_{M}\right)
\end{aligned}
$$


где $q_{j} \in \mathbb{R} \forall j=1, \ldots, M, \varphi\left(q_{1}, \ldots, q_{M}\right) \in L^{2}\left(\mathbb{R}^{M}\right), A_{j k}=A_{k j}^{*}$, и $D_{j k}=D_{k j}, \forall j, k=$ $1, \ldots, M$. А именно, если собственные значения системы уравнений в вариациях $\beta_{l}, l=$ $1, \ldots, M$, действительны, то решения уравнения (6) имеют следующий вид

$$
\begin{aligned}
\lambda_{\{\nu\}}= & \sum_{j=1}^{M}\left(\beta_{j}\left(k_{j}+\frac{1}{2}\right)-\frac{1}{2} A_{j j}\right)+\frac{1}{2} \sum_{j=1}^{M} \sum_{k=1}^{M}\left(q_{j}^{0} q_{k}^{0}\left(A_{j k}+D_{j k}+D_{j k}^{*}\right)\right. \\
& \left.+p_{j}^{0} p_{k}^{0}\left(A_{j k}-D_{j k}-D_{j k}^{*}\right) q_{j}^{0} q_{k}^{0}\left(i A_{j k}-i A_{k j}+2 i D_{j k}^{*}-2 i D_{j k}\right)\right) \\
& +\frac{1}{\sqrt{2}} \sum_{j=1}^{M}\left(q_{j}^{0}\left(f_{j}+f_{j}^{*}\right)+p_{j}^{0}\left(i f_{j}^{+}-i f_{j}\right)\right), \\
\varphi_{\nu}\left(q_{1}, \ldots, q_{M}\right)= & \exp \left(\frac{i}{\hbar} \sum_{j=1}^{M} p_{j}^{0}\left(q_{j}-q_{j}^{0}\right)\right. \\
& \left.+\frac{i}{2 \hbar} \sum_{j=1}^{M} \sum_{k=1}^{M}\left(q_{j}-q_{j}^{0}\right)\left(B C^{-1}\right)_{j k}\left(q_{k}-q_{k}^{0}\right)\right) \prod_{l=1}^{M} \bar{\Lambda}_{l} e^{\nu e} \cdot 1,
\end{aligned}
$$

где $\{\nu\}$ - набор целых чисел, $\nu_{j} \geqslant 0, j=1, \ldots, M$, a $q_{j}^{0}, p_{j}^{0}$ являются решениями системы уравнений:

$$
\begin{aligned}
& \sum_{k=1}^{M}\left(q_{k}^{0}\left(A_{j k}+A_{k j}+2 D_{j k}+2 D_{j k}^{*}\right)+p_{k}^{0}\left(i A_{j k}-i A_{k j}-2 i D_{j k}+2 i D_{j k}^{*}\right)\right) \\
& \quad+\sqrt{2}\left(f_{j}+f_{j}^{*}\right)=0, \\
& \sum_{k=1}^{M}\left(q_{k}^{0}\left(i A_{j k}-i A_{k j}+2 i D_{j k}^{*}-2 i D_{j k}\right)+p_{k}^{0}\left(A_{j k}+A_{k j}-2 D_{j k}-2 D_{j k}^{*}\right)\right) \\
& \quad+i \sqrt{2}\left(f_{j}^{*}-f_{j}\right)=0 ;
\end{aligned}
$$

$\bar{\Lambda}_{l}, l=1, \ldots, M,-$ дифференциальные операторы вида

$$
\bar{\Lambda}_{l}=\sum_{m=1}^{M}\left(\frac{\sqrt{\hbar}}{i} C_{m l}^{*} \frac{\partial}{\partial q_{m}}-\frac{1}{\sqrt{\hbar}}\left(B^{*}-B C^{-1} C^{*}\right)_{m l}\left(q_{m}-q_{m}^{0}\right)\right)
$$

где $\beta_{j}, j=1, \ldots, M$, и матрицы $B$ и $C$ являются решениями следующей системы уравнений (система уравнений в вариациях):

$$
\begin{aligned}
i \beta_{l} C_{j l}= & \frac{1}{2} \sum_{k=1}^{M} B_{k l}\left(A_{j k}+A_{k j}-2 D_{j k}-2 i D_{j k}^{*}\right) \\
& +\frac{1}{2} \sum_{k=1}^{M} C_{k l}\left(i A_{k j}-i A_{j k}+2 i D_{j k}^{*}-2 i D_{j k}\right), \\
i \beta_{l} B_{j l}=- & \frac{1}{2} \sum_{k=1}^{M} C_{k l}\left(A_{j k}+A_{k j}+2 D_{j k}+2 D_{j k}^{*}\right) \\
& -\frac{1}{2} \sum_{k=1}^{M} B_{k l}\left(i A_{j k}-i A_{k j}+2 i D_{j k}^{*}-2 i D_{j k}\right),
\end{aligned}
$$




$$
\sum_{l=1}^{M}\left(B_{l j} C_{l k}^{*}-C_{l j} B_{l k}^{*}\right)=2 i \delta_{j k}, \quad \sum_{l=1}^{M}\left(B_{l j} C_{l k}-C_{l j} B_{l k}\right)=0
$$

Уравнение (3) является бесконечномерным аналогом уравнения (6). Применим для его решения формулы конечномерного случая. Мы получим из (7), что термы $\lambda\left(x_{1}, p_{1} ; \ldots ; x_{N}, p_{N}\right)$ имеют следующий вид

$$
\begin{aligned}
& \lambda\{m\}\left(x_{1}, p_{1} ; \ldots ; x_{N}, p_{N}\right)=\sum_{j=1}^{N} H_{0}\left(x_{j}, p_{j}\right) \\
& +\frac{1}{2} \sum_{j=1}^{N} \sum_{k=1}^{N} V\left(x_{j}, p_{j} ; x_{k}, p_{k}\right)+\sum_{\eta} \beta_{\eta}\left(m_{\eta}+\frac{1}{2}\right)-\frac{1}{2} \sum_{\alpha=1}^{N_{B}} \sum_{\vec{n}} \Omega_{\alpha}\left(\frac{2 \pi}{L} \vec{n}\right) \\
& -\frac{1}{2} \sum_{\alpha=1}^{N_{B}} \sum_{\vec{n}} \sum_{j=1}^{N} A_{\alpha \alpha}\left(\frac{2 \pi}{L} \vec{n}, \frac{2 \pi}{L} \vec{n}^{\prime} ; x_{j}, p_{j}\right) \\
& +\frac{1}{2} \sum_{\alpha=1}^{N_{B}} \Omega_{\alpha}\left(\frac{2 \pi}{L} \vec{n}\right)\left(\left(Q_{\alpha, \vec{n}}^{0}\right)^{2}+\left(\mathcal{P}_{\alpha, \vec{n}}^{0}\right)^{2}\right) \\
& +\frac{1}{2} \sum_{\alpha=1}^{N_{B}} \sum_{\beta=1}^{N_{B}} \sum_{\vec{n}} \sum_{\vec{n}^{\prime}} \sum_{j=1}^{N}\left(Q _ { \alpha , \vec { n } } ^ { 0 } Q _ { \beta , \vec { n } ^ { \prime } } ^ { 0 } \left(A_{\alpha \beta}\left(\frac{2 \pi}{L} \vec{n}, \frac{2 \pi}{L} \vec{n}^{\prime} ; x_{j}, p_{j}\right)\right.\right. \\
& \left.+B_{\alpha \beta}\left(\frac{2 \pi}{L} \vec{n}, \frac{2 \pi}{L} \vec{n}^{\prime} ; x_{j}, p_{j}\right)+B_{\alpha \beta}^{*}\left(\frac{2 \pi}{L} \vec{n}, \frac{2 \pi}{L} \vec{n}^{\prime} ; x_{j}, p_{j}\right)\right) \\
& +\mathcal{P}_{\alpha, \vec{n}}^{0} \mathcal{P}_{\beta, \vec{n}^{\prime}}^{0}\left(A_{\alpha \beta}\left(\frac{2 \pi}{L} \vec{n}, \frac{2 \pi}{L} \vec{n}^{\prime} ; x_{j}, p_{j}\right)-B_{\alpha \beta}\left(\frac{2 \pi}{L} \vec{n}, \frac{2 \pi}{L} \vec{n}^{\prime} ; x_{j}, p_{j}\right)\right. \\
& \left.-B_{\alpha \beta}^{*}\left(\frac{2 \pi}{L} \vec{n}, \frac{2 \pi}{L} \vec{n}^{\prime} ; x_{j}, p_{j}\right)\right) \\
& +Q_{\alpha, \vec{n}}^{0} \mathcal{P}_{\beta, \vec{n}^{\prime}}^{0}\left(i A_{\alpha \beta}\left(\frac{2 \pi}{L} \vec{n}, \frac{2 \pi}{L} \vec{n}^{\prime} ; x_{j}, p_{j}\right)-i A_{\beta \alpha}\left(\frac{2 \pi}{L} \vec{n}, \frac{2 \pi}{L} \vec{n}^{\prime} ; x_{j}, p_{j}\right)\right. \\
& \left.\left.+2 i B_{\alpha \beta}^{*}\left(\frac{2 \pi}{L} \vec{n}, \frac{2 \pi}{L} \vec{n}^{\prime} ; x_{j}, p_{j}\right)-2 i B_{\alpha \beta}\left(\frac{2 \pi}{L} \vec{n}, \frac{2 \pi}{L} \vec{n}^{\prime} ; x_{j}, p_{j}\right)\right)\right) \\
& +\frac{1}{\sqrt{2}} \sum_{\alpha=1}^{N_{B}} \sum_{\vec{n}} \sum_{j=1}^{N}\left(Q_{\alpha, \vec{n}}^{0}\left(f_{\alpha}\left(\frac{2 \pi}{L} ; x_{j}, p_{j}\right)+f_{\alpha}^{*}\left(\frac{2 \pi}{L} ; x_{j}, p_{j}\right)\right)\right. \\
& \left.+\mathcal{P}_{\alpha, \vec{n}}\left(f_{\alpha}^{*}\left(\frac{2 \pi}{L} ; x_{j}, p_{j}\right)-i f_{\alpha}\left(\frac{2 \pi}{L} ; x_{j}, p_{j}\right)\right)\right),
\end{aligned}
$$

где $\{m\}$ набор целых чисел, $m_{\eta} \geqslant 0, \eta=1, \ldots, \infty$, аргументы $\left(x_{1}, p_{1}, \ldots, ; x_{N}, p_{N}\right)$ у $\beta_{\eta}$ и $Q_{\alpha, \vec{n}}^{0}, \mathcal{P}_{\alpha, \vec{n}}^{0}$ опушены, а $Q_{\alpha, \vec{n}}^{0}, \mathcal{P}_{\alpha, \vec{n}}^{0}$ являются решениями системы уравнений вида:

$$
\Omega_{\alpha}\left(\frac{2 \pi}{L} \vec{n}\right) Q_{\alpha, \vec{n}}^{0}+\frac{1}{2} \sum_{\beta=1}^{N_{B}} \sum_{\vec{n}^{\prime}} \sum_{j=1}^{N}\left(Q _ { \beta , \vec { n } ^ { \prime } } ^ { 0 } \left(A_{\alpha \beta}\left(\frac{2 \pi}{L} \vec{n}, \frac{2 \pi}{L} \vec{n}^{\prime} ; x_{j}, p_{j}\right)\right.\right.
$$




$$
\begin{aligned}
& +2 B_{\alpha \beta}\left(\frac{2 \pi}{L} \vec{n}, \frac{2 \pi}{L} \vec{n}^{\prime} ; x_{j}, p_{j}\right) \\
& \left.+2 B_{\alpha \beta}^{*}\left(\frac{2 \pi}{L} \vec{n}, \frac{2 \pi}{L} \vec{n}^{\prime} ; x_{j}, p_{j}\right)+A_{\beta \alpha}\left(\frac{2 \pi}{L} \vec{n}, \frac{2 \pi}{L} \vec{n}^{\prime} ; x_{j}, p_{j}\right)\right) \\
& +\mathcal{P}_{\beta, \vec{n}^{\prime}}^{0}\left(i A_{\alpha \beta}\left(\frac{2 \pi}{L} \vec{n}, \frac{2 \pi}{L} \vec{n}^{\prime} ; x_{j}, p_{j}\right)-i A_{\beta \alpha}\left(\frac{2 \pi}{L} \vec{n}, \frac{2 \pi}{L} \vec{n}^{\prime} ; x_{j}, p_{j}\right)\right. \\
& \left.\left.+2 i B_{\alpha \beta}^{*}\left(\frac{2 \pi}{L} \vec{n}, \frac{2 \pi}{L} \vec{n}^{\prime} ; x_{j}, p_{j}\right)-2 i B_{\alpha \beta}\left(\frac{2 \pi}{L} \vec{n}, \frac{2 \pi}{L} \vec{n}^{\prime} ; x_{j}, p_{j}\right)\right)\right) \\
& +\frac{1}{\sqrt{2}} \sum_{j=1}^{N}\left(f_{\alpha}\left(\frac{2 \pi}{L} \vec{n} ; x_{j}, p_{j}\right)+f_{\alpha}^{*}\left(\frac{2 \pi}{L} \vec{n} ; x_{j}, p_{j}\right)\right)=0, \\
& \Omega_{\alpha}\left(\frac{2 \pi}{L} \vec{n}\right) \mathcal{P}_{\alpha, \vec{n}}^{0}+\frac{1}{2} \sum_{\beta=1}^{N_{B}} \sum_{\vec{n}^{\prime}} \sum_{j=1}^{N}\left(\mathcal { P } _ { \beta , \vec { n } ^ { \prime } } ^ { 0 } \left(A_{\alpha \beta}\left(\frac{2 \pi}{L} \vec{n}, \frac{2 \pi}{L} \vec{n}^{\prime} ; x_{j}, p_{j}\right)\right.\right. \\
& +A_{\beta \alpha}\left(\frac{2 \pi}{L} \vec{n}, \frac{2 \pi}{L} \vec{n}^{\prime} ; x_{j}, p_{j}\right) \\
& \left.-2 B_{\alpha \beta}\left(\frac{2 \pi}{L} \vec{n}, \frac{2 \pi}{L} \vec{n}^{\prime} ; x_{j}, p_{j}\right)-2 B_{\beta \alpha}^{*}\left(\frac{2 \pi}{L} \vec{n}, \frac{2 \pi}{L} \vec{n}^{\prime} ; x_{j}, p_{j}\right)\right) \\
& +Q_{\beta, \vec{n}^{\prime}}^{0}\left(i A_{\beta \alpha}\left(\frac{2 \pi}{L} \vec{n}, \frac{2 \pi}{L} \vec{n}^{\prime} ; x_{j}, p_{j}\right)-i A_{\alpha \beta}\left(\frac{2 \pi}{L} \vec{n}, \frac{2 \pi}{L} \vec{n}^{\prime} ; x_{j}, p_{j}\right)\right. \\
& \left.\left.+2 i B_{\alpha \beta}^{*}\left(\frac{2 \pi}{L} \vec{n}, \frac{2 \pi}{L} \vec{n}^{\prime} ; x_{j}, p_{j}\right)-2 i B_{\alpha \beta}\left(\frac{2 \pi}{L} \vec{n}, \frac{2 \pi}{L} \vec{n}^{\prime} ; x_{j}, p_{j}\right)\right)\right) \\
& +\frac{1}{\sqrt{2}} \sum_{j=1}^{N} i\left(f_{\alpha}^{*}\left(\frac{2 \pi}{L} \vec{n} ; x_{j}, p_{j}\right)-f_{\alpha}\left(\frac{2 \pi}{L} \vec{n} ; x_{j}, p_{j}\right)\right)=0
\end{aligned}
$$

$\beta_{\eta}$ являются решениями системы уравнений вида:

$$
\begin{aligned}
i \beta_{\eta} Z_{\alpha, \vec{n}}^{\eta}= & \Omega_{\alpha}\left(\frac{2 \pi}{L} \vec{n}\right) W_{\alpha, \vec{n}}^{\eta}+\frac{1}{2} \sum_{\beta=1}^{N_{B}} \sum_{\vec{n}^{\prime}} \sum_{j=1}^{N}\left(W _ { \beta , \vec { n } ^ { \prime } } ^ { \eta } \left(A_{\alpha \beta}\left(\frac{2 \pi}{L} \vec{n}, \frac{2 \pi}{L} \vec{n}^{\prime} ; x_{j}, p_{j}\right)\right.\right. \\
& +A_{\beta \alpha}\left(\frac{2 \pi}{L} \vec{n}, \frac{2 \pi}{L} \vec{n}^{\prime} ; x_{j}, p_{j}\right)-2 B_{\alpha \beta}\left(\frac{2 \pi}{L} \vec{n}, \frac{2 \pi}{L} \vec{n}^{\prime} ; x_{j}, p_{j}\right) \\
& \left.-2 B_{\alpha \beta}^{*}\left(\frac{2 \pi}{L} \vec{n}, \frac{2 \pi}{L} \vec{n}^{\prime} ; x_{j}, p_{j}\right)\right) \\
& +Z_{\beta, \vec{n}^{\prime}}^{\eta}\left(i A_{\beta \alpha}\left(\frac{2 \pi}{L} \vec{n}, \frac{2 \pi}{L} \vec{n}^{\prime} ; x_{j}, p_{j}\right)-i A_{\alpha \beta}\left(\frac{2 \pi}{L} \vec{n}, \frac{2 \pi}{L} \vec{n}^{\prime} ; x_{j}, p_{j}\right)\right. \\
& \left.\left.+2 i B_{\alpha \beta}^{*}\left(\frac{2 \pi}{L} \vec{n}, \frac{2 \pi}{L} \vec{n}^{\prime} ; x_{j}, p_{j}\right)-2 i B_{\alpha \beta}\left(\frac{2 \pi}{L} \vec{n}, \frac{2 \pi}{L} \vec{n}^{\prime} ; x_{j}, p_{j}\right)\right)\right),
\end{aligned}
$$




$$
\begin{aligned}
-i \beta_{\eta} W_{\alpha, \vec{n}}^{\eta}= & \Omega_{\alpha}\left(\frac{2 \pi}{L} \vec{n}\right) Z_{\alpha, \vec{n}}^{\eta}+\frac{1}{2} \sum_{\beta=1}^{N_{B}} \sum_{\vec{n}^{\prime}} \sum_{j=1}^{N}\left(Z _ { \beta , \vec { n } ^ { \prime } } ^ { \eta } \left(A_{\alpha \beta}\left(\frac{2 \pi}{L} \vec{n}, \frac{2 \pi}{L} \vec{n}^{\prime} ; x_{j}, p_{j}\right)\right.\right. \\
& +A_{\beta \alpha}\left(\frac{2 \pi}{L} \vec{n}, \frac{2 \pi}{L} \vec{n}^{\prime} ; x_{j}, p_{j}\right)+2 B_{\alpha \beta}\left(\frac{2 \pi}{L} \vec{n}, \frac{2 \pi}{L} \vec{n}^{\prime} ; x_{j}, p_{j}\right) \\
& \left.+2 B_{\alpha \beta}^{*}\left(\frac{2 \pi}{L} \vec{n}, \frac{2 \pi}{L} \vec{n}^{\prime} ; x_{j}, p_{j}\right)\right) \\
& +W_{\beta, \vec{n}^{\prime}}^{\eta}\left(i A_{\alpha \beta}\left(\frac{2 \pi}{L} \vec{n}, \frac{2 \pi}{L} \vec{n}^{\prime} ; x_{j}, p_{j}\right)-i A_{\beta \alpha}\left(\frac{2 \pi}{L} \vec{n}, \frac{2 \pi}{L} \vec{n}^{\prime} ; x_{j}, p_{j}\right)\right. \\
& \left.\left.+2 i B_{\alpha \beta}^{*}\left(\frac{2 \pi}{L} \vec{n}, \frac{2 \pi}{L} \vec{n}^{\prime} ; x_{j}, p_{j}\right)-2 i B_{\alpha \beta}\left(\frac{2 \pi}{L} \vec{n}, \frac{2 \pi}{L} \vec{n}^{\prime} ; x_{j}, p_{j}\right)\right)\right) \\
& \sum_{\alpha=1}^{N_{B}} \sum_{\vec{n}}\left(W_{\alpha, \vec{n}}^{\eta}\left(Z_{\alpha, \vec{n}}^{\eta^{\prime}}\right)^{*}-Z_{\alpha, \vec{n}}^{\eta}\left(W_{\alpha, \vec{n}}^{\eta^{\prime}}\right)^{*}\right)=2 i \delta_{\eta \eta^{\prime}}, \\
& \sum_{\alpha=1}^{N_{B}} \sum_{\vec{n}}\left(W_{\alpha, \vec{n}}^{\eta} Z_{\alpha, \vec{n}}^{\eta^{\prime}}-W_{\alpha, \vec{n}}^{\eta} Z_{\alpha, \vec{n}}^{\eta^{\prime}}\right)=0, \quad \forall \eta, \eta^{\prime}=1, \ldots, \infty .
\end{aligned}
$$

Здесь и в дальнейшем опущены аргументы $\left(x_{1}, p_{1} ; \ldots ; x_{N}, p_{N}\right)$ у $\beta_{\eta}, Q_{\alpha, \vec{n}}^{0}, \mathcal{P}_{\alpha, \vec{n}}^{0}, Z_{\alpha, \vec{n}}^{\eta}$, $W_{\alpha, \vec{n}}^{\eta}$

Пусть функции $Q_{\alpha}(q) \in C^{\infty}\left(\mathbb{R}^{3}\right), \mathcal{P}_{\alpha}(q) \in C^{\infty}\left(\mathbb{R}^{3}\right), Z_{\alpha}^{\eta}(q) \in C^{\infty}\left(\mathbb{R}^{3}\right)$ и $W_{\alpha}^{\eta}(q) \in$ $C^{\infty}\left(\mathbb{R}^{3}\right) \forall \alpha=1, \ldots, N$, являются решениями следующих уравнений

$$
\begin{aligned}
& \Omega_{\alpha}(q) Q_{\alpha}(q)+\frac{1}{2} \sum_{\beta=1}^{N_{B}} \sum_{\vec{n}^{\prime}} \sum_{j=1}^{N}\left(A_{\alpha \beta}\left(\stackrel{2}{q}, \frac{2 \pi}{L} \vec{n}^{\prime} ; x_{j}, p_{j}\right)+A_{\beta \alpha}\left(\frac{2 \pi}{L} \vec{n}^{\prime}, \stackrel{2}{q} ; x_{j}, p_{j}\right)\right. \\
& \left.+2 B_{\alpha \beta}\left(\stackrel{2}{q}, \frac{2 \pi}{L} \vec{n}^{\prime} ; x_{j}, p_{j}\right)+2 B_{\alpha \beta}^{*}\left(\stackrel{2}{q}, \frac{2 \pi}{L} \vec{n}^{\prime} ; x_{j}, p_{j}\right)\right) \exp \left(\left(\frac{2 \pi}{L} n^{\prime}-\stackrel{2}{q}\right) \frac{1}{\partial q}\right) Q_{\beta}(q) \\
& +\frac{1}{2} \sum_{\beta=1}^{N_{B}} \sum_{\vec{n}^{\prime}} \sum_{j=1}^{N}\left(i A_{\alpha \beta}\left(\stackrel{2}{q}, \frac{2 \pi}{L} \vec{n}^{\prime} ; x_{j}, p_{j}\right)-i A_{\beta \alpha}\left(\frac{2 \pi}{L} \vec{n}^{\prime}, \stackrel{2}{q} ; x_{j}, p_{j}\right)\right. \\
& \left.+2 i B_{\alpha \beta}^{*}\left(\stackrel{2}{q}, \frac{2 \pi}{L} \vec{n}^{\prime} ; x_{j}, p_{j}\right)-2 i B_{\alpha \beta}\left(\stackrel{2}{q}, \frac{2 \pi}{L} \vec{n}^{\prime} ; x_{j}, p_{j}\right)\right) \exp \left(\left(\frac{2 \pi}{L} n^{\prime}-\stackrel{2}{q}\right) \frac{\stackrel{\partial}{\partial}}{\partial q}\right) \mathcal{P}_{\beta}(q) \\
& +\frac{1}{\sqrt{2} \sum_{j=1}^{N}}\left(f_{\alpha}\left(q ; x_{j}, p_{j}\right)+f_{\alpha}^{*}\left(q ; x_{j}, p_{j}\right)\right)=0 \\
& \Omega_{\alpha}(q) \mathcal{P}_{\alpha}(q)+\frac{1}{2} \sum_{\beta=1}^{N_{B}} \sum_{\vec{n}^{\prime}} \sum_{j=1}^{N}\left(A_{\alpha \beta}\left(\stackrel{2}{q}, \frac{2 \pi}{L} \vec{n}^{\prime} ; x_{j}, p_{j}\right)+A_{\beta \alpha}\left(\frac{2 \pi}{L} \vec{n}^{\prime}, \stackrel{2}{q} ; x_{j}, p_{j}\right)\right. \\
& \left.-2 B_{\alpha \beta}\left(\stackrel{2}{q}, \frac{2 \pi}{L} \vec{n}^{\prime} ; x_{j}, p_{j}\right)-2 B_{\alpha \beta}^{*}\left(\stackrel{2}{q}, \frac{2 \pi}{L} \vec{n}^{\prime} ; x_{j}, p_{j}\right)\right) \exp \left(\left(\frac{2 \pi}{L} n^{\prime}-\stackrel{2}{q}\right) \frac{\partial}{\partial q}\right) \mathcal{P}_{\beta}(q) \\
& +\frac{1}{2} \sum_{\beta=1}^{N_{B}} \sum_{\vec{n}^{\prime}} \sum_{j=1}^{N}\left(i A_{\beta \alpha}\left(\frac{2 \pi}{L} \vec{n}^{\prime}, \stackrel{2}{q} ; x_{j}, p_{j}\right)-i A_{\alpha \beta}\left(\stackrel{2}{q}, \frac{2 \pi}{L} \vec{n}^{\prime} ; x_{j}, p_{j}\right)\right.
\end{aligned}
$$




$$
\begin{aligned}
& \left.+2 i B_{\alpha \beta}^{*}\left(\stackrel{2}{q}, \frac{2 \pi}{L} \vec{n}^{\prime} ; x_{j}, p_{j}\right)-2 i B_{\alpha \beta}\left(\stackrel{2}{q}, \frac{2 \pi}{L} \vec{n}^{\prime} ; x_{j}, p_{j}\right)\right) \exp \left(\left(\frac{2 \pi}{L} n^{\prime}-\stackrel{2}{q}\right) \frac{\stackrel{\partial}{\partial}}{\partial q}\right) Q_{\beta}(q) \\
& +\frac{1}{\sqrt{2}} \sum_{j=1}^{N}\left(f_{\alpha}^{*}\left(q ; x_{j}, p_{j}\right)-f_{\alpha}\left(q ; x_{j}, p_{j}\right)\right)=0
\end{aligned}
$$

а также

$$
\begin{aligned}
& i \beta_{\eta} Z_{\alpha}^{\eta}(q)=\Omega_{\alpha}(q) W_{\alpha}^{\eta}(q)+\frac{1}{2} \sum_{\beta=1}^{N_{B}} \sum_{\vec{n}^{\prime}} \sum_{j=1}^{N}\left(A_{\alpha \beta}\left(\stackrel{2}{q}, \frac{2 \pi}{L} \vec{n}^{\prime} ; x_{j}, p_{j}\right)\right. \\
& +A_{\beta \alpha}\left(\frac{2 \pi}{L} \vec{n}^{\prime}, \stackrel{2}{q} ; x_{j}, p_{j}\right)-2 B_{\alpha \beta}\left(\stackrel{2}{q}, \frac{2 \pi}{L} \vec{n}^{\prime} ; x_{j}, p_{j}\right) \\
& \left.-2 B_{\alpha \beta}^{*}\left(\stackrel{2}{q}, \frac{2 \pi}{L} \vec{n}^{\prime} ; x_{j}, p_{j}\right)\right) \exp \left(\left(\frac{2 \pi}{L} n^{\prime}-\stackrel{2}{q}\right) \frac{1}{\partial} \frac{\partial}{\partial q}\right) W_{\beta}^{\eta}(q) \\
& +\frac{1}{2} \sum_{\beta=1}^{N_{B}} \sum_{\vec{n}^{\prime}} \sum_{j=1}^{N}\left(i A_{\beta \alpha}\left(\frac{2 \pi}{L} \vec{n}^{\prime}, \stackrel{2}{q} ; x_{j}, p_{j}\right)\right. \\
& -i A_{\alpha \beta}\left(\stackrel{2}{q}, \frac{2 \pi}{L} \vec{n}^{\prime} ; x_{j}, p_{j}\right)+2 i B_{\alpha \beta}^{*}\left(\stackrel{2}{q}, \frac{2 \pi}{L} \vec{n}^{\prime} ; x_{j}, p_{j}\right) \\
& \left.-2 i B_{\alpha \beta}\left(\stackrel{2}{q}, \frac{2 \pi}{L} \vec{n}^{\prime} ; x_{j}, p_{j}\right)\right) \exp \left(\left(\frac{2 \pi}{L} n^{\prime}-\stackrel{2}{q}\right) \frac{1}{\partial q}\right) Z_{\beta}^{\eta}(q), \\
& -i \beta_{\eta} W_{\alpha}^{\eta}(q)=\Omega_{\alpha}(q) Z_{\alpha}^{\eta}(q)+\frac{1}{2} \sum_{\beta=1}^{N_{B}} \sum_{\vec{n}^{\prime}} \sum_{j=1}^{N}\left(A_{\alpha \beta}\left(\stackrel{2}{q}, \frac{2 \pi}{L} \vec{n}^{\prime} ; x_{j}, p_{j}\right)\right. \\
& +A_{\beta \alpha}\left(\frac{2 \pi}{L} \vec{n}^{\prime}, \stackrel{2}{q} ; x_{j}, p_{j}\right)+2 B_{\alpha \beta}\left(\stackrel{2}{q}, \frac{2 \pi}{L} \vec{n}^{\prime} ; x_{j}, p_{j}\right) \\
& \left.+2 B_{\alpha \beta}^{*}\left(\stackrel{2}{q}, \frac{2 \pi}{L} \vec{n}^{\prime} ; x_{j}, p_{j}\right)\right) \exp \left(\left(\frac{2 \pi}{L} n^{\prime}-\stackrel{2}{q}\right) \frac{1}{\partial q}\right) Z_{\beta}^{\eta}(q) \\
& +\frac{1}{2} \sum_{\beta=1}^{N_{B}} \sum_{\vec{n}^{\prime}} \sum_{j=1}^{N}\left(i A_{\alpha \beta}\left(\stackrel{2}{q}, \frac{2 \pi}{L} \vec{n}^{\prime} ; x_{j}, p_{j}\right)\right. \\
& -i A_{\beta \alpha}\left(\frac{2 \pi}{L} \vec{n}^{\prime}, \stackrel{2}{q} ; x_{j}, p_{j}\right)+2 i B_{\alpha \beta}^{*}\left(\stackrel{2}{q}, \frac{2 \pi}{L} \vec{n}^{\prime} ; x_{j}, p_{j}\right) \\
& \left.-2 i B_{\alpha \beta}\left(\stackrel{2}{q}, \frac{2 \pi}{L} \vec{n}^{\prime} ; x_{j}, p_{j}\right)\right) \exp \left(\left(\frac{2 \pi}{L} n^{\prime}-\stackrel{2}{q}\right) \frac{1}{\partial q}\right) W_{\beta}^{\eta}(q),
\end{aligned}
$$

где $q \in \mathbb{R}^{3}$, число над оператором обозначает порядок его действия, и

$$
\left(\frac{2 \pi}{L} n^{\prime}-q\right) \frac{\partial}{\partial q}=\sum_{j=1}^{3}\left(\frac{2 \pi}{L} n_{j}^{\prime}-q_{j}\right) \frac{\partial}{\partial q_{j}}
$$


Решения уравнений (15) и (16) определяют решения уравнений (13) и (14) следующего вида

$$
\begin{array}{ll}
Q_{\alpha, \vec{n}}^{0}=Q_{\alpha}\left(\frac{2 \pi}{L} \vec{n}\right), & \mathcal{P}_{\alpha, \vec{n}}^{0}=\mathcal{P}_{\alpha}\left(\frac{2 \pi}{L} \vec{n}\right) \\
Z_{\alpha, \vec{n}}^{\eta}=Z_{\alpha}^{\eta}\left(\frac{2 \pi}{L} \vec{n}\right), & W_{\alpha, \vec{n}}^{\eta}=W_{\alpha}^{\eta}\left(\frac{2 \pi}{L} \vec{n}\right)
\end{array}
$$

Уравнение (4) является уравнением переноса [1] для следующего уравнения Шрёдингера

$$
\begin{aligned}
& i \hbar \frac{\partial \varphi_{\nu}}{\partial t}\left(x_{1}, s_{1} ; \ldots ; x_{N}, s_{N} ; t\right)=\lambda\left(x_{1}^{2},-\hbar \frac{1}{\partial x_{1}} ; \ldots ; x_{N}^{2},-\hbar \frac{1}{\partial x_{N}}\right) \varphi_{\nu}\left(x_{1}, s_{1} ; \ldots ; x_{N}, s_{N} ; t\right) \\
& -i \hbar \sum_{\mu=1}^{K} G_{\nu \mu}\left(\stackrel{2}{x}_{1},-\hbar \frac{\partial}{\partial x_{1}} ; \ldots ; x_{N}^{2},-\hbar \frac{\partial}{\partial x_{N}}\right) \varphi_{\mu}\left(x_{1}, s_{1} ; \ldots ; x_{N}, s_{N} ; t\right)+O\left(\hbar^{2}\right), \\
& \nu=1, \ldots, K,
\end{aligned}
$$

Нашей задачей является нахождение асимптотики решения уравнений (1) по двум параметрам: сильному взаимодействию и большому числу фермионов, так чтобы получить сверхпроводимость при сильном взаимодействии. В силу определения сверхпроводимости, данного автором с помощью понятия спектральных серий, возможность такой двойной асимптотики зависит от оценки элементов матрищы перехода из состояния определяемого одной серией и другой серией.

Оказьвается, что туннельная асимптотика [1] позволяет оценить вероятности перехода и они оказьваются мальми одновременно и равномерно по двум вьшеуказанным параметрам.

С другой стороны, квазиклассическая асимптотика и оценки невязки в $L_{2}\left(\mathbb{R}^{N}\right)$ позволяют считать число фермионов $N \sim 1 / \sqrt{h}$ (где под “ $h$ " понимается безразмерньй параметр, зависящий от константы взаимодействия). Поэтому мы получаем, что наша асимптотика совпадает с асимптотикой уравнения Шрёдингера (17), уравнением переноса для которого является уравнение (4).

К уравнению Шрёдингера (17) мы можем применить теорию, развитую в [3], тем самым получив температуру фазового перехода и величину скачка теплоемкости для исследуемых задач. Поэтому по $\bmod (1 / N)$ мы можем применить развитую ранее теорию [3] к уравнению (17).

Аналогично [3] мы можем написать уравнения для определения фазового перехода для частного случая, когда $G=0$, а терм $\lambda$ представляет из себя сумму по парным взаимодействиям с заданным $\hbar$-псевдо-дифференциальным оператором

$$
V\left(x-y,-i \hbar \frac{\partial}{\partial x},-i \hbar \frac{\partial}{\partial y}, \hbar\right),
$$

где квантование понимается по Вейлю. Пусть трансформация Фурье

$$
\widetilde{V}\left(k, p_{1}, p_{2}, \hbar\right)=\int d \xi V\left(\xi, p_{1}, p_{2}, \hbar\right) e^{i k \xi / \hbar}
$$


удовлетворяет условиям

$$
\widetilde{V}\left(k, p_{1}, p_{2}, \hbar\right)=\overline{\widetilde{V}}\left(k, p_{1}, p_{2}, \hbar\right)=\widetilde{V}\left(-k, p_{2}, p_{1}, \hbar\right)
$$

Рассмотрим случай, когда $x$ принадлежит трехмерному тору с радиусом $L$. Из $(u-v)$-трансформации Боголюбова и метода осреднения [3] мыполучим следующие уравнения для $G_{p}$ и $R_{p}$ :

$$
\begin{aligned}
-i \hbar \frac{\partial}{\partial t} G_{p}= & \sum_{q} \tilde{V}(-p+q, q,-q, \hbar)\left(R_{p}^{*} R_{q}-R_{q}^{*} R_{p}\right), \\
i \hbar \frac{\partial}{\partial t} R_{p}= & R_{p}\left(\frac{p^{2}}{m}+\sum_{q} G_{q}(\widetilde{V}(0, p, q, \hbar)-\widetilde{V}(p-q, p, q, \hbar)\right. \\
& +\widetilde{V}(0,-p, q, \hbar)-\widetilde{V}(-p-q,-p, q, \hbar))) \\
& +\sum_{q} \tilde{V}(-p+q, q,-q)\left(1-G_{p}-G_{-p}\right) R_{q},
\end{aligned}
$$

где $p, q \in(2 \pi \hbar / L) \mathbb{Z}^{3}$. Функции $G_{p}$ и $R_{p}$ удовлетворяют условиям

$$
\begin{gathered}
G_{p}=G_{p}^{*}, \quad R_{p}=R_{-p} \\
G_{p}=G_{p}^{2}+\left|R_{p}\right|^{2} .
\end{gathered}
$$

Обозначим

$$
\mathbf{A}_{p}=\left(\begin{array}{cc}
G_{p}-1 / 2 & R_{p}^{*} \\
R_{p} & -G_{p}+1 / 2
\end{array}\right), \quad \mathbf{L}_{p}=\left(\begin{array}{cc}
A_{p} & B_{p}^{*} \\
B_{p} & -A_{-p}
\end{array}\right)
$$

где

$$
\begin{aligned}
& A_{p}=\frac{p^{2}}{2 m}-E+\widetilde{V}(0) \sum_{q} G_{q}-\sum_{q} \tilde{V}(p-q) G_{q} \\
& B_{p}=\sum_{q} \tilde{V}(p-q) R_{q} .
\end{aligned}
$$

Несложно убедиться, что коммутатор матриц $\mathbf{A}_{p}$ и $\mathbf{L}_{p}$ имеет вид

$$
\left[\mathbf{A}_{p}, \mathbf{L}_{p}\right]=\left(\begin{array}{cc}
\alpha_{p} & -\beta_{p}^{*} \\
\beta_{p} & -\alpha_{p}^{*}
\end{array}\right)
$$

где $\alpha_{p}$ - левая часть уравнения (18a), а $\beta_{p}$ - левая часть уравнения (18б).

Согласно пуантилистическому постулату, $\mathbf{A}_{p}=f\left(\mathbf{L}_{p}\right)$, где

$$
f(\xi)=\frac{1}{e^{\xi / \theta}+1}-\frac{1}{2}
$$


В силу того, что матрица $\mathbf{L}_{p}^{2}$ диагональна, матрица $\mathbf{A}_{p}$ представима в виде $\mathbf{A}_{p}=$ $f\left(\lambda_{p}\right) \mathbf{L}_{p} / \lambda_{p}$, где

$$
\lambda_{p}=\sqrt{A_{p}^{2}+\left|B_{p}\right|^{2}}
$$

- собственное число $\mathbf{L}_{p}$. Приравнивая элементы матриц $\mathbf{A}_{p}$ и $f\left(\lambda_{p}\right) \mathbf{L}_{p} / \lambda_{p}$, получим уравнения

$$
\begin{gathered}
G_{p}=\frac{f\left(\lambda_{p}\right)}{\lambda_{p}}\left(\frac{p^{2}}{2 m}-E+\widetilde{V}(0) \sum_{q} G_{q}-\sum_{q} \tilde{V}(p-q) G_{q}\right)+\frac{1}{2} \\
R_{p}=\frac{f\left(\lambda_{p}\right)}{\lambda_{p}} \sum_{q} \tilde{V}(p-q) R_{q} .
\end{gathered}
$$

Рассмотрим теперь несколько примеров.

ПРИмеР 1. Взаимодействие әлектронов с фононами в ионных кристаллах [4].

Пусть в гамильтониане $(1) H_{0}(x, p ; \hbar)=p^{2} / 2 m$, где $m$ - эффективная масса электрона в кристалле, $V\left(x_{1}, p_{1} ; x_{2}, p_{2} ; \hbar\right)=0, N_{B}=1, \Omega_{1}(q)=\Omega$ - постоянная; $A_{11}\left(q, q^{\prime}\right.$; $x, p ; \hbar)=B_{11}\left(q, q^{\prime} ; x, p ; \hbar\right)=0, f_{1}(q ; x, p ; \hbar)=\left(1 / \sqrt{L^{3}}\right) e^{-i q x}(g /|q|), f_{1}(0 ; x, p ; \hbar)$ при $q \neq 0$, где $g$ - постоянная. В этом случае (1) отвечает системе электронов, взаимодействующих с фононами. Уравнения (15) примут вид

$$
\begin{aligned}
& \Omega Q(q)+\sqrt{\frac{2}{L^{3}}} \frac{g}{|q|} \sum_{j=1}^{N} \cos q x_{j}=0 \\
& \Omega \mathcal{P}(q)-\sqrt{\frac{2}{L^{3}}} \frac{g}{|q|} \sum_{j=1}^{N} \sin q x_{j}=0 .
\end{aligned}
$$

Уравнения (16) примут вид

$$
\begin{gathered}
i \beta_{\eta} Z^{\eta}(q)=\Omega W^{\eta}(q), \quad-i \beta_{\eta} W^{\eta}(q)=\Omega Z^{\eta}(q) \\
\sum_{\vec{n}}\left(W^{\eta}\left(\frac{2 \pi}{L} \vec{n}\right)\left(Z^{\eta^{\prime}}\left(\frac{2 \pi}{L} \vec{n}\right)\right)^{*}-Z^{\eta}\left(\frac{2 \pi}{L} \vec{n}\right)\left(W^{\eta^{\prime}}\left(\frac{2 \pi}{L} \vec{n}\right)\right)^{*}\right)=2 i \delta_{\eta \eta^{\prime}} \\
\sum_{\vec{n}}\left(W^{\eta}\left(\frac{2 \pi}{L} \vec{n}\right) Z^{\eta^{\prime}}\left(\frac{2 \pi}{L} \vec{n}\right)-W^{\eta^{\prime}}\left(\frac{2 \pi}{L} \vec{n}\right) Z^{\eta}\left(\frac{2 \pi}{L} \vec{n}\right)\right)=0 .
\end{gathered}
$$

Из уравнений $(27)$ и (28) получим, что термы $\lambda\left(x_{1}, p_{1} ; \ldots ; x_{N}, p_{N}\right)$ имеют следующий вид

$$
\begin{aligned}
\lambda_{M}\left(x_{1}, p_{1} ; \ldots ; x_{N}, p_{N}\right)= & \sum_{j=1}^{N} \frac{p_{j}^{2}}{2 m}+\Omega M \\
& -\frac{g^{2}}{\Omega L^{3}} \sum_{j=1}^{N} \sum_{k=1, k \neq j}^{N} \sum_{\vec{n}} \frac{\exp \left\{i \frac{2 \pi}{L} \vec{n}\left(x_{j}-x_{k}\right)\right\}}{\left|\frac{2 \pi}{L} \vec{n}\right|^{2}}+C,
\end{aligned}
$$


где

$$
C=-\frac{g^{2} N}{\Omega L^{3}} \sum_{\vec{n}} \frac{1}{\left|\frac{2 \pi}{L} \vec{n}\right|^{2}}
$$

- постоянная и $M=0, \ldots, \infty$ - целое число, нумерующее термы. Матрица $G$ равна нулю для всех $M$. Уравнение (17), отвечающее данному случаю, может быть представлено в виде $(19)$, где $T(p ; \hbar)=p^{2} / 2 m$, a $W\left(x_{1}-x_{2} ; p_{1}, p_{2} ; \hbar\right)$ в пределе при $L \rightarrow \infty$ имеет вид $W\left(x_{1}-x_{2} ; p_{1}, p_{2} ; \hbar\right) \rightarrow-g^{2} / 2 \pi \Omega\left|x_{2}-x_{2}\right|$.

ПримеР 2. Модель Фрелиха [5].

Пусть в гамильтониане $(1) H_{0}(x, p ; \hbar)=p^{2} / 2 m$, где $m$ - эффективная масса электрона в кристалле, $V\left(x_{1}, p_{1} ; x_{2}, p_{2} ; \hbar\right)=0, N_{B}=1$,

$$
\Omega_{1}(q)= \begin{cases}c_{\text {sound }}(q) & \text { для }|q|<\omega_{D} / c_{\text {sound }}, \\ 0 & \text { для }|q| \geqslant \omega_{D} / c_{\text {sound }},\end{cases}
$$

$A_{11}\left(q, q^{\prime} ; x, p ; \hbar\right)=B_{11}\left(q, q^{\prime} ; x, p ; \hbar\right)=0, f_{1}(q ; x, p ; \hbar)=g \sqrt{\Omega_{1}(q) / 2 L^{3}} \exp \{-i q x\}$, где $g$-постоянная, $c_{\text {sound }}-$ скорость звука, a $\omega_{D}$ - частота Дебая. В этом случае гамильтониан (1) отвечает модели Фрелиха, которая описьвает взаимодействие электронов и акустических фононов. Уравнения (15) примут вид

$$
\begin{aligned}
& \Omega_{1}(q) Q(q)+g \sqrt{\frac{\Omega_{1}(q)}{L^{3}}} \sum_{j=1}^{N} \cos q x_{j}=0 \\
& \Omega_{1}(q) \mathcal{P}(q)-g \sqrt{\frac{\Omega_{1}(q)}{L^{3}}} \sum_{j=1}^{N} \sin q x_{j}=0 .
\end{aligned}
$$

Уравнения (16) примут вид

$$
\begin{gathered}
i \beta_{\eta} Z^{\eta}(q)=\Omega_{1} W^{\eta}(q), \quad-i \beta_{\eta} W^{\eta}(q)=\Omega_{1} Z^{\eta}(q), \\
\sum_{\vec{n}}\left(W^{\eta}\left(\frac{2 \pi}{L} \vec{n}\right)\left(Z^{\eta^{\prime}}\left(\frac{2 \pi}{L} \vec{n}\right)\right)^{*}-Z^{\eta}\left(\frac{2 \pi}{L} \vec{n}\right)\left(W^{\eta^{\prime}}\left(\frac{2 \pi}{L} \vec{n}\right)\right)^{*}\right)=2 i \delta_{\eta \eta^{\prime}} \\
\sum_{\vec{n}}\left(W^{\eta}\left(\frac{2 \pi}{L} \vec{n}\right) Z^{\eta^{\prime}}\left(\frac{2 \pi}{L} \vec{n}\right)-W^{\eta^{\prime}}\left(\frac{2 \pi}{L} \vec{n}\right) Z^{\eta}\left(\frac{2 \pi}{L} \vec{n}\right)\right)=0 .
\end{gathered}
$$

Решив уравнения (30) и (31), получим, что термы $\lambda\left(x_{1}, p_{1} ; \ldots ; x_{N}, p_{N}\right)$ для модели $\Phi$ релиха имеют вид

$$
\begin{aligned}
& \lambda_{\{m\}}\left(x_{1}, p_{1} ; \ldots ; x_{N}, p_{N}\right)=\sum_{j=1}^{N} \frac{p_{j}^{2}}{2 m}+\sum_{\vec{n}} \Omega_{1}\left(\frac{2 \pi}{L} \vec{n}\right) m_{\vec{n}} \\
&-\frac{g^{2}}{2 L^{3}} \sum_{j=1}^{N} \sum_{k=1}^{N} \sum_{n,|\vec{n}|<(L / 2 \pi)\left(\omega_{D} / c_{\text {sound }}\right)} \exp \left\{i \frac{2 \pi}{L} n\left(x_{j}-x_{k}\right)\right\},
\end{aligned}
$$

где $\{m\}$ - наборы целых чисел $m_{\vec{n}}=0, \ldots, \infty$ для $|\vec{n}|<(L / 2 \pi)\left(\omega_{D} / c_{\text {sound }}\right)$. В данном случае матрища $G$ равна нулю для всех термов. Уравнение (17) представимо в виде 
уравнения Шрёдингера, где кинетическая энергия равна $p^{2} / 2 m$, а потенциал взаимодействия имеет вид

$$
V\left(x_{1}-x_{2} ; p_{1}, p_{2} ; \hbar\right)=-\frac{g^{2}}{L^{3}} \sum_{\vec{n}} \exp \left\{i \frac{2 \pi}{L} n\left(x_{1}-x_{2}\right)\right\} .
$$

В пределе при $L \rightarrow \infty$ этот потенциал примет вид

$$
V\left(x_{1}-x_{2} ; p_{1}, p_{2} ; \hbar\right)=-\frac{g^{2}}{2 \pi^{2}|x|^{2}}\left(\frac{\omega_{D}}{c_{\text {sound }}} \omega s\left(\frac{\omega_{D}}{c_{\text {sound }}}|x|\right)-\frac{1}{|x|} \sin \left(\frac{\omega_{D}}{c_{\text {sound }}}|x|\right)\right)
$$

где $x=x_{1}-x_{2}$.

ПримеР 3. Электрическое поле и әлектроны.

Рассмотрим систему электронов, которые взаимодействуют с электрическим полем. Будем считать, что магнитного поля в системе нет. Гамильтониан такой системы имеет вид $(1)$, где $H_{0}(x, p ; \hbar)=p^{2} / 2 m, m$ - масса электрона, $V\left(x_{1}, p_{;} x_{2}, p_{2} ; \hbar\right)=0, N_{B}=1$, $\Omega_{1}(q)=-c|q|, A_{11}\left(q, q^{\prime} ; x, p ; \hbar\right)=B_{11}\left(q, q^{\prime} ; x, p ; \hbar\right)=0, f_{1}(q)=e \sqrt{c /|q|} e^{-i q x} \sqrt{2 \pi / L^{3}}$, $c$ - скорость света, а $e$ - заряд электрона.

Для данной модели уравнения (15) и (16) принимают вид, аналогичньй уравнениям (30) и (31) соответственно. Термы в данном случае имеют вид

$$
\begin{aligned}
\lambda_{\{m\}}\left(x_{1}, p_{s} ; \ldots ; x_{N}, p_{N}\right)= & \sum_{j=1}^{N} \frac{p_{j}^{2}}{2 m}-\sum_{\vec{n}} c \frac{2 \pi}{L}|\vec{n}| m_{\vec{n}} \\
& +e^{2} \frac{2 \pi}{L^{3}} \sum_{j=1}^{N} \sum_{k=1}^{N} \sum_{\vec{n}} \exp \left\{i \frac{2 \pi}{L} n\left(x_{j}-x_{k}\right)\right\} \frac{1}{\left|\frac{2 \pi}{L} \vec{n}\right|^{2}}
\end{aligned}
$$

где $\left\{m_{\vec{n}}\right\}$ - набор целых чисел таких, что $m_{\vec{n}} \geqslant 0$ и матрица $G$ для всех термов равна нулю. Потенциал парного взаимодействия для данного случая представляет собой закон Кулона

$$
V\left(x_{1}-x_{2} ; p_{1}, p_{2}\right)=\frac{e^{2}}{\left|x_{1}-x_{2}\right|}
$$

ПрИмеР 4. Взаимодействие әлектронов с квантовым әлектромагнитным полем в калибровке Кулона [6].

В квантовой электродинамике в кулоновской калибровке гамильтониан электронов и электромагнитного поля имеет вид [6]:

$$
\begin{aligned}
& H=\sum_{j=1}^{N} \frac{p_{j}^{2}}{2 m}+\sum_{\vec{n}} \sum_{\sigma=1}^{2} c\left|\frac{2 \pi}{L} \vec{n}\right| \widehat{b}_{\sigma, \vec{n}}^{+} \widehat{b}_{\sigma, \vec{n}}^{-} \\
& -\frac{e}{m c} \sum_{j=1}^{N} \sum_{\vec{n}} \sum_{\sigma=1}^{2} \sqrt{\frac{2 \pi c}{L^{3}\left|\frac{2 \pi}{L} \vec{n}\right|}}\left(p_{j}, e_{n, \sigma}\right)\left(e^{i \frac{2 \pi}{L} n x_{j}} \widehat{b}_{\sigma, \vec{n}}^{-}+e^{-i \frac{2 \pi}{L} n x_{j}} \widehat{b}_{\sigma, \vec{n}}^{+}\right) \\
& +\frac{e^{2}}{2 m c^{2}} \sum_{j=1}^{N} \sum_{\vec{n}} \sum_{\vec{n}^{\prime}} \sum_{\sigma=1}^{2} \sum_{\sigma^{\prime}=1}^{2} \frac{2 \pi c}{L^{3} \sqrt{\left|\frac{2 \pi}{L} \vec{n}\right|\left|\frac{2 \pi}{L} \vec{n}^{\prime}\right|}}\left(e_{n, \sigma}, e_{n^{\prime}, \sigma^{\prime}}\right) \\
& \times\left(e^{i \frac{2 \pi}{L} n x_{j}} \widehat{b}_{\sigma, \vec{n}}^{-}+e^{-i \frac{2 \pi}{L} n x_{j}} \widehat{b}_{\sigma, \vec{n}}^{+}\right)\left(e^{i \frac{2 \pi}{L} n^{\prime} x_{j}} \widehat{b}_{\sigma, \vec{n}^{\prime}}^{-}+e^{-i \frac{2 \pi}{L} n^{\prime} x_{j}} \widehat{b}_{\sigma, \vec{n}^{\prime}}^{+}\right),
\end{aligned}
$$


где $c$ - скорость света, $e$ - заряд электрона, $m$ - масса электрона, $\widehat{b}_{\sigma, \vec{n}}^{ \pm}$- операторы, удовлетворяюшие коммутационным соотношениям

$$
\left[\widehat{b}_{\sigma, \vec{n}}^{-}, \widehat{b}_{\sigma^{\prime}, \vec{n}^{\prime}}^{+}\right]=\delta_{\sigma \sigma^{\prime}} \delta_{\vec{n}, \vec{n}^{\prime}}, \quad\left[\widehat{b}_{\sigma, \vec{n}}^{ \pm}, \widehat{b}_{\sigma^{\prime}, \vec{n}^{\prime}}^{ \pm}\right]=0
$$

где $e_{n, \sigma}, \sigma=1,2,-$ ортонормированньй базис векторов на плоскости в трехмерном пространстве, которая перпендикулярна $\vec{n}$. В данном случае $A$ и $B$ не равны нулю. Это обстоятельство усложняет вычисления. Если пренебречь в гамильтониане (32)последним членом, что отвечает малости магнитного поля, то термы в пределе при $L \rightarrow \infty$ имеют следующий вид

$$
\begin{aligned}
\lambda_{\{m\}}\left(x_{1}, p_{1} ; \ldots ; x_{N}, p_{N}\right)=\sum_{j=1}^{N} \frac{p_{j}^{2}}{2 m}+\sum_{\vec{n}} \sum_{\sigma=1}^{2} c\left|\frac{2 \pi}{L} \vec{n}\right| m_{\vec{n}, \sigma} \\
-\frac{e^{2}}{4 m^{2} c^{2}} \sum_{j=1}^{N} \sum_{k=1}^{N} \frac{1}{\left|x_{j}-x_{k}\right|}\left(\left(p_{j}, p_{k}\right)+\frac{\left(p_{j}, x_{j}-x_{k}\right)\left(p_{k}, x_{j}-x_{k}\right)}{\left|x_{j}-x_{k}\right|^{2}}\right) .
\end{aligned}
$$

Вычисление матрицы $G$ очень сложно, и его не удается провести в явном виде, даже при отбрасывании последнего члена в (32). Если учитывать последний член в (32) по теории возмущений, то в первом порядке добавок к (33) в пределе при $L \rightarrow \infty$ имеет вид

$$
\begin{aligned}
\delta \lambda_{\{m\}} & \left(x_{1}, p_{1} ; \ldots ; x_{N}, p_{N}\right)=-\frac{e^{4}}{8 m^{3} c^{4}} \sum_{j=1}^{N} \sum_{k=1}^{N} \sum_{l=1}^{N} \frac{1}{\left|x_{j}-x_{k}\right|} \frac{1}{\left|x_{j}-x_{l}\right|} \\
& \times\left(p_{k}+\frac{\left(p_{k}, x_{j}-x_{k}\right)}{\left|x_{j}-x_{k}\right|^{2}}\left(x_{j}-x_{k}\right)\right)\left(p_{l}+\frac{\left(p_{l}, x_{j}-x_{l}\right)}{\left|x_{j}-x_{l}\right|^{2}}\left(x_{j}-x_{l}\right)\right) .
\end{aligned}
$$

Как видим, в выражении (34) присутствуют члены с тройным взаимодействием между электронами.

ПримеР 5. Модель Хаббарда. Рассмотрим систему фермионов и бозонов на двумерной дискретной решетке, гамильтониан которой в представлении вторичного квантования имеет вид [7]

$$
\widehat{H}=\sum_{\vec{q}} \omega(\vec{q}) \widehat{\alpha}_{\vec{q}}^{+} \widehat{\alpha}_{\vec{q}}^{-}+\frac{z t}{\sqrt{L}} \sum_{\vec{k} \vec{q}}\left(M(\vec{k} \vec{q}) \widehat{h}_{\vec{k}}^{+} \widehat{h}_{\vec{k}-\vec{q}}^{-} \widehat{\alpha}_{\vec{q}}^{-}+M^{*}(\vec{k} \vec{q}) \widehat{\alpha}_{\vec{q}}^{+} \widehat{h}_{\vec{k}-\vec{q}}^{+} \widehat{h}_{\vec{k}}^{-}\right)
$$

где $z, t$-постоянные [7], $L$ - число узлов квадратной решетки такое, что $\sqrt{L}=N$-целое число, $\vec{k}=(2 \pi / N)\left(k_{1}, k_{2}\right), \vec{q}=(2 \pi / N)\left(q_{1}, q_{2}\right)$, где $k_{1}, k_{2}, q_{1}, q_{2}=0,1, \ldots, N-1, \widehat{\alpha}_{\vec{q}}^{+}, \widehat{\alpha}_{\vec{q}}^{-}$ - бозонные операторы рождения и уничтожения, $\widehat{h}_{\vec{k}}^{+}, \widehat{h}_{\vec{k}}^{-}$- фермионные операторы рождения и уничтожения, $\omega(q) \in C^{\infty}\left(\mathbb{R}^{2}\right), M\left(q_{1}, q_{2}\right) \in C^{\infty}\left(\mathbb{R}^{4}\right)$, и введено обозначение

$$
\sum_{\vec{q}} \cdots=\sum_{q_{1}=0}^{N-1} \sum_{q_{2}=0}^{N-1} \cdots
$$

Если функция $M(k, q)$ зависит также от $N$ следующим образом

$$
M(k, q)=\sqrt{N} F(k, \sqrt{N} q),
$$


где $F(k, q) \in C^{\infty}\left(\mathbb{R}^{4}\right)$, то в пределе при $N \rightarrow \infty$ бозонные переменные можно исключить развитым вьше методом. Для $n$ фермионов получим гамильтониан:

$$
H\left(x_{1}, p_{1} ; \ldots ; x_{n}, p_{n}\right)=-\frac{z^{2} t^{2}}{2(2 \pi)^{2}} \sum_{s=1}^{n} \sum_{r=1}^{n} V\left(x_{s}, p_{s} ; x_{r}, p_{r}\right)
$$

где $x_{j}$ принадлежит двумерному тору со стороной, равной $1, p_{j} \in \mathbb{R}^{2}$, и $V\left(x_{1}, p_{1} ; x_{2}, p_{2}\right)$ имеет вид

$$
\begin{aligned}
V\left(x_{1}, p_{1} ; x_{2}, p_{2}\right)= & \iint_{-\infty}^{+\infty} d z_{1} d z_{2} \cdot \frac{1}{\omega(0)}\left[F\left(p_{1}, z\right) F^{*}\left(p_{2}, z\right) e^{i z\left(x_{1}-x_{2}\right)}\right. \\
& \left.+F\left(p_{2}, z\right) F^{*}\left(p_{1}, z\right) e^{i z\left(x_{2}-x_{1}\right)}\right] .
\end{aligned}
$$

Автор приносит глубокую благодарность Г. В. Ковалю за большую помощь в вычислениях.

\section{СПИСОК ЦИТИРОВАННОЙ ЛИТЕРАТУРЫ}

[1] Маслов В.П. Теория возмущений. М.: Изд-во МГУ, 1965.

[2] Маслов В.П. Операторные методы. М.: Наука, 1973.

[3] Maslov V.P. Quasi-particles associated with isoenergetic manifolds corresponding to classical self-consistent fields. X // Russian J. Math. Phys. 1997. V. 5. № 2. P. 273-278.

[4] Давыдов А. С. Теория твердого тела. М.: Наука, 1976.

[5] Займан Дж. Электроны и фононы. М.: ИЛ, 1962.

[6] Хакен Х. Квантополевая теория твердого тела. М.: Наука, 1980.

[7] Плакида Н. М. Высокотемпературные сверхпроводники. М.: Международная программа образования, 1996.

Московский государственный универсистет им. М.В.Ломоносова

Поступило 10.07.1999 